\title{
Exercise modulation of tumour perfusion and hypoxia to improve radiotherapy response in prostate cancer
}

\author{
Oliver Schumacher $\mathbb{1}^{1,2} \cdot$ Daniel A. Galvão $0^{1,2} \cdot$ Dennis R. Taaffe ${ }^{1,2,3} \cdot$ Raphael Chee ${ }^{1,2,4} \cdot$ Nigel Spry ${ }^{1,2,4} \cdot$ \\ Robert U. Newton $\mathbb{D}^{1,2,3}$
}

Received: 30 March 2020 / Revised: 25 May 2020 / Accepted: 24 June 2020 / Published online: 6 July 2020

(c) The Author(s) 2020. This article is published with open access

\begin{abstract}
Background An increasing number of studies indicate that exercise plays an important role in the overall care of prostate cancer (PCa) patients before, during and after treatment. Historically, research has focused on exercise as a modulator of physical function, psychosocial well-being as well as a countermeasure to cancer- and treatment-related adverse effects. However, recent studies reveal that exercise may also directly influence tumour physiology that could beneficially affect the response to radiotherapy.

Methods In this narrative review, we provide an overview of tumour vascular characteristics that limit the effect of radiation and establish a rationale for exercise as adjunct therapy during PCa radiotherapy. Further, we summarise the existing literature on exercise as a modulator of tumour perfusion and hypoxia and outline potential future research directions.

Results Preclinical research has shown that exercise can reduce intratumoral hypoxia-a major limiting factor in radiotherapy - by improving tumour perfusion and vascularisation. In addition, preliminary evidence suggests that exercise training can improve radiotherapy treatment outcomes by increasing natural killer cell infiltration in a murine PCa model. Conclusions Exercise is a potentially promising adjunct therapy for men with $\mathrm{PCa}$ undergoing radiotherapy that may increase its effectiveness. However, exercise-induced tumour radiosensitisation remains to be confirmed in preclinical and clinical trials, as does the optimal exercise prescription to elicit such effects.
\end{abstract}

\section{Introduction}

Radiotherapy is a cornerstone in the management of cancer and used to treat a myriad of malignancies, including prostate. Approximately $50 \%$ of all cancer patients receive radiotherapy during the course of their treatment for either curative or palliative purposes [1]. Moreover, $\sim 40 \%$ of prostate cancer $(\mathrm{PCa})$ patients aged 65 and above receive radiotherapy as their primary treatment [2]. Despite ever

Oliver Schumacher

o.schumacher@ecu.edu.au

1 Exercise Medicine Research Institute, Edith Cowan University, Joondalup, WA, Australia

2 School of Medical and Health Sciences, Edith Cowan University, Joondalup, WA, Australia

3 School of Human Movement and Nutrition Sciences, University of Queensland, Brisbane, QLD, Australia

4 GenesisCare, Joondalup, WA, Australia evolving technological advances that allow for more precise targeting of tumours, treatment resistance and subsequent disease recurrence pose a significant challenge in the field of radiation oncology [3-5].

Approximately $20-39 \%$ of patients with localised PCa treated with external beam radiotherapy experience local disease persistence and subsequent recurrence with an increased risk of developing distant metastases [6-8]. The hypoxic tumour microenvironment (TME) is recognised as a major limiting factor in the treatment of cancer and is an established prognostic marker that has been associated with treatment resistance as well as local disease persistence and recurrence $[9,10]$. Moreover, hypoxia is a common feature of many solid tumours, including PCa [11].

While exercise has been associated with a reduced risk of $\mathrm{PCa}$ progression and mortality [12], little is known about the underlying biological mechanisms contributing to these benefits. It has been postulated that exercise may acutely reduce tumour hypoxia through increased perfusion of tumour tissue and that structural adaptations of the tumour vasculature in response to exercise training may potentially 
result in a less aggressive tumour and further enhance treatment response [13].

Given the significance of hypoxia in compromising cancer treatment, the purpose of this narrative review is to: (1) provide an overview of the characteristics of tumour vasculature and how they affect tumour hypoxia; (2) establish a rationale for exercise as adjunct therapy during cancer treatment; (3) summarise the existing literature on exercise as a modulator of tumour perfusion and hypoxia; and (4) outline potential future research directions.

\section{Tumour vasculature and hypoxia}

Angiogenesis is a normal physiological process that occurs in situations like wound healing or in response to exercise training $[14,15]$. In tumours, however, rapid proliferation of cells results in the formation of hypoxic areas and subsequent persistent local and unbalanced expression of proand anti-angiogenic factors leads to the formation of dysfunctional and structurally abnormal vascular networks, which are a characteristic feature of the TME [16]. These vascular networks are often very disorganised, tortuous and immature, which results in heterogeneous and variable tumour blood flow, further aggravating rather than alleviating hypoxia [17, 18]. Consequently, hypoxic and thus angiogenic signalling are sustained, further driving abnormal tumour vascularisation. In addition, tumour blood vessels are leakier than normal blood vessels, resulting in increased interstitial fluid pressure (IFP) [19]. Increased IFP can lead to sudden, intermittent perfusion changes as a result of collapsing blood vessels, which, in turn, limits oxygen delivery to the tumour, further worsening tumour hypoxia and decreasing treatment efficacy [20]. Moreover, increased vascular permeability plays a key role in metastasis by facilitating intravasation of cancer cells [21].

Hypoxia arising from vascular abnormalities can be differentiated into two forms - chronic and acute [22, 23]. Chronic hypoxia arises when the distance between blood vessels and tumour cells surpass the diffusion distance of oxygen. Chronic hypoxia is therefore also known as diffusion-limited hypoxia. When oxygen diffuses from blood vessels into the adjacent tissue it is consumed by the tumour cells closest to the perfused vessel, creating a gradient in the partial pressure of oxygen $\left(\mathrm{PO}_{2}\right)$. The decrease in $\mathrm{PO}_{2}$ with increasing distance from the perfused vessel renders tumour cells further away from this vessel more hypoxic. Acute hypoxia, on the other hand, is caused by limited and varying perfusion of blood vessels supplying tumour cells (i.e. perfusion-limited hypoxia). As a result, all cells that are normally supplied by a now transiently compromised blood vessel are temporarily, or acutely, hypoxic. The reasons for acutely limited perfusion are numerous and include occlusion of vessels with blood or circulating tumour cells, collapse of vessels due to high IFP, and spontaneous vasoconstriction in normal tumour vessels that then affect downstream tumour vessels [22, 23]. In addition to poor tumour perfusion and oxygen diffusion, anaemia is a further major cause of tumour hypoxia (i.e. anaemic hypoxia) that results from cancer- and treatment-associated reduced oxygen transport capacity of the blood [24, 25].

With decreasing tissue oxygen levels, tumour cells become increasingly resistant to ionising radiation due to reduced availability of oxygen to stabilise DNA strand breaks caused by radiation and reactive oxygen species $[23,26]$. In PCa, hypoxia is associated with a more aggressive tumour phenotype, increased metastasis, and patients with more hypoxic tumours have a poorer prognosis compared to patients with a less hypoxic TME [27]. Milosevic et al. [28] directly measured tumour hypoxia with a transrectal needle-electrode technique in 247 patients with localised PCa before radiotherapy and found that hypoxia was associated with early biochemical failure and local disease recurrence. Intrinsic markers of tumour hypoxia (i.e. hypoxia-inducible factor 1-alpha) have also been investigated and increased expression identified patients with shorter time to biochemical failure independent of clinical tumour stage, biopsy Gleason score, serum prostate-specific antigen concentration, and radiotherapy dose [29]. Targeting and reversing tumour hypoxia thus has been proposed as a potential mechanism to improve treatment response to radiotherapy and enhance survival [27].

Pharmacological interventions aimed at inducing vascular normalisation to improve treatment efficacy through increased drug delivery and tumour oxygenation have primarily focused on suppressing vascular endothelial growth factor (VEGF) signalling given its key role in angiogenesis [30]. Anti-angiogenic (or anti-VEGF) therapy, originally developed to disrupt a tumour's blood supply and deprive it of oxygen and nutrients, has shown some promise in normalising tumour vasculature when given in low doses, but has thus far not been tested in prospective clinical trials to permit concrete conclusions [30, 31]. Furthermore, tumour vascular normalisation has a small therapeutic window, is complicated by tumour heterogeneity, and associated with significant side effects as well as malignant progression [32-35]. Hence, the use of pharmacological angiogenesis inhibitors has not gained wide clinical application. On the other hand, mild hyperthermia is increasingly being explored as a radiosensitiser for its effects on tumour vasculature and DNA repair mechanisms [36]. Relatively low temperatures of $39-42{ }^{\circ} \mathrm{C}$ have been shown to increase tumour perfusion, thus decreasing hypoxia and enhancing tumour radiosensitivity [36]. In a recent study using an orthotopic PCa mouse model, hyperthermia significantly slowed tumour growth and resulted in significantly smaller 
Fig. 1 Potential tumour physiology at rest (left side) and during exercise (right side). a Acute exercise increases tumour blood flow and tumour vascular resistance is reduced.

b Augmented tumour perfusion pressure as a result of increased tumour blood flow may lead to greater oxygen $\left(\mathrm{O}_{2}\right)$ diffusion distance and thus reduce diffusion-limited hypoxic tumour areas. c Tumour vasculature is dysfunctional and structurally abnormal, resulting in heterogeneous and variable tumour blood flow. Exercise increases the area of tumour perfusion and therefore reduces perfusion-limited hypoxia. Created with BioRender.com.

A

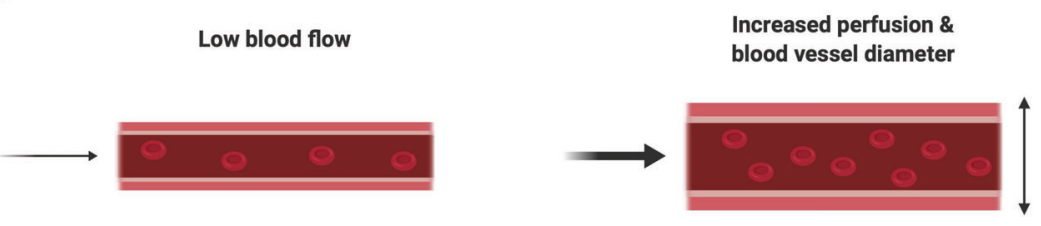

B

Short $\mathrm{O}_{2}$ diffusion distance

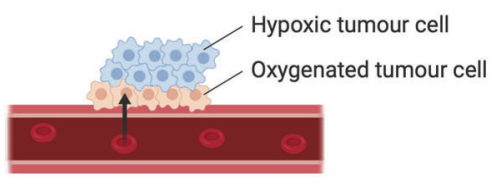

C

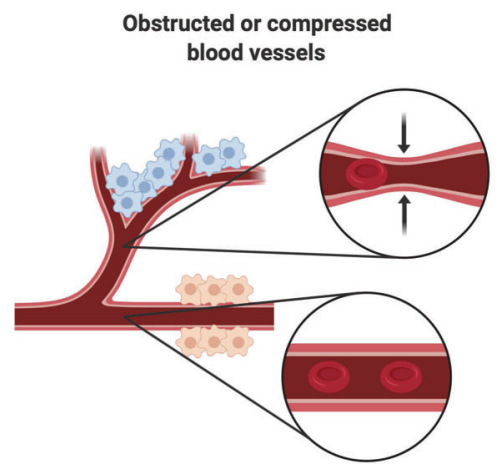

Increased perfusion pressure \& $\mathrm{O}_{2}$ diffusion distance

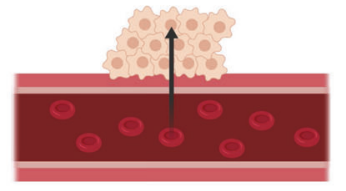

Increased number of patent \& perfused blood vessels

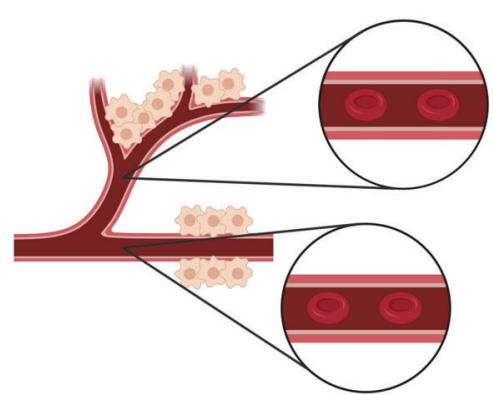

normalised nadir tumour volumes compared to radiotherapy without hyperthermia [37]. Similar to anti-VEGF therapy, though, the timing and dosing of hyperthermia to achieve the desired radiosensitising effect is complex and makes clinical application challenging [36].

\section{Exercise as adjunct therapy in cancer treatment}

In the field of exercise oncology, i.e. the application of exercise as medicine in the oncology setting, there is consistent evidence that greater levels of physical activity, commonly defined as 'any bodily movement produced by skeletal muscles that results in energy expenditure' [38], are associated with a lower risk of developing certain types of cancer as well as decreased all-cause and cancer-specific mortality for patients diagnosed with breast, colorectal, or PCa [39]. For example, Kenfield et al. [40] found that men with $\mathrm{PCa}$ who did $\geq 3 \mathrm{~h} /$ week of vigorous physical activity had a $49 \%$ lower risk of all-cause mortality and a $61 \%$ lower risk of dying from PCa compared to men who did $<1$ $\mathrm{h}$ /week of vigorous physical activity. Furthermore, Richman et al. [41] reported that men with localised PCa who walked at a brisk pace for $\geq 3 \mathrm{~h} /$ week had a $57 \%$ lower risk of disease progression compared to men who walked at an easy pace for $<3 \mathrm{~h} /$ week. This association between walking pace and reduced risk of cancer progression was independent of walking duration [41], suggesting that not only the volume of physical activity but also the intensity of physical activity is an important factor regarding clinical outcomes in PCa. It is important to note, however, that these findings are based on observational data and randomised controlled trials are currently investigating the impact of exercise on overall survival in patients with PCa as well as colon cancer [42, 43].

In addition to reduced risk of disease progression and mortality, physical activity and exercise, defined as 'a subset of physical activity that is planned, structured, and repetitive and has as a final or an intermediate objective the improvement or maintenance of physical fitness' [38], are increasingly being recognised as an important part of treatment during active therapy to improve or maintain physical function and quality of life in cancer patients [44]. Research has consistently demonstrated that exercise improves physical and mental health in men with $\mathrm{PCa}$ during and following completion of therapeutic interventions and can reduce the level of fatigue experienced by 
Fig. 2 Potential mechanism of exercise-modulated tumour perfusion and hypoxia.

Systemic effects of augmented cardiac output and increased arterial pressure resulting from mild hyperthermia and increased stroke volume and heart rate in response to acute exercise (top panel) may influence local physiological responses and vascular adaptation in the tumour microenvironment (bottom panel). Improved tumour perfusion, either as a result of increased systemic blood flow during exercise or as a result of vascular maturation, may enhance acute and chronic oxygenation of hypoxic tumour areas, thus resulting in improved radiotherapy efficacy.

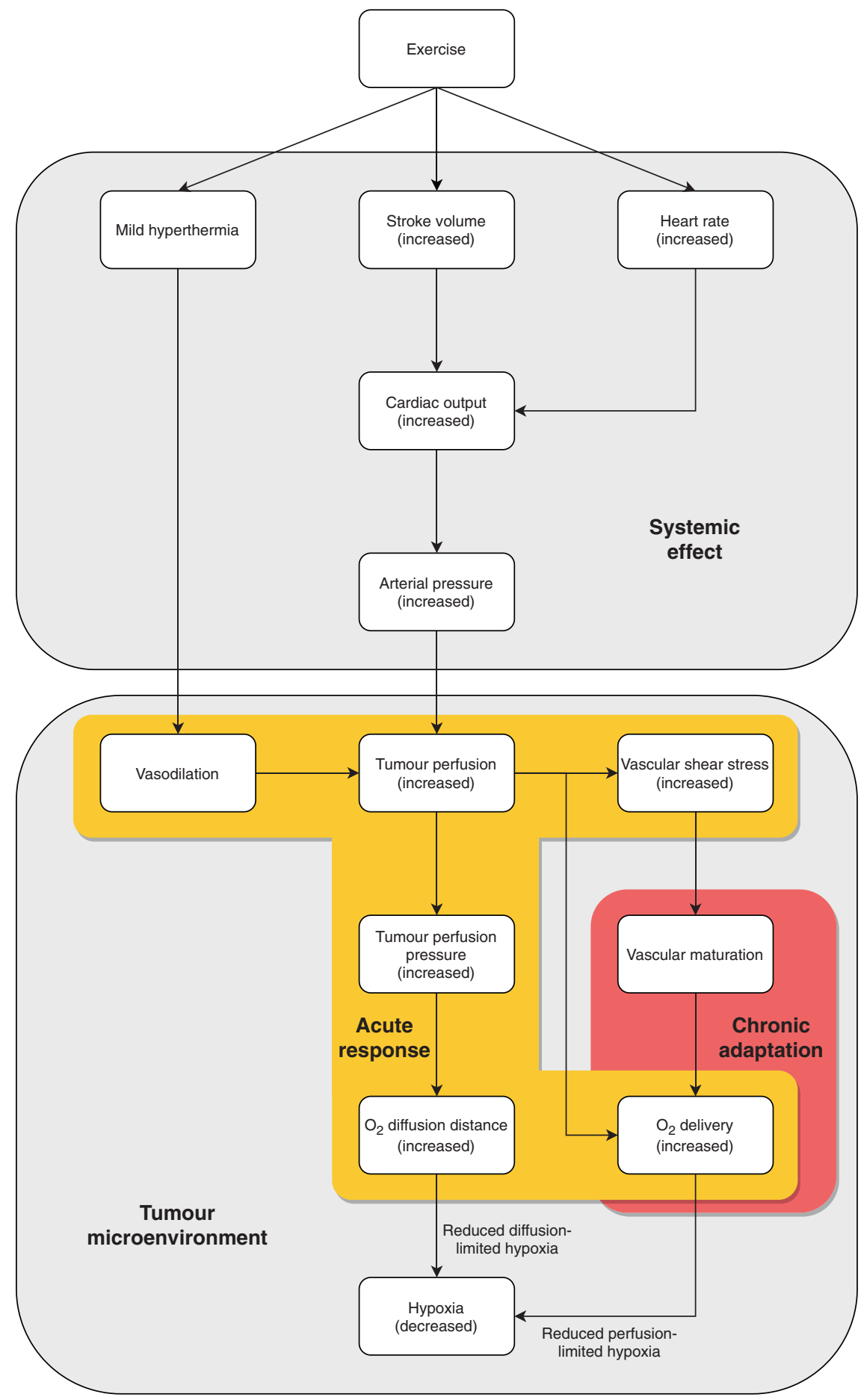

many patients [45-48]. Some studies have also indicated that exercise may help alleviate toxicities associated with treatment $[49,50]$.

More recently, the role of exercise as an adjunct therapy to enhance the effectiveness of conventional cancer treatments such as radiotherapy is gaining a strong interest for its effects of modulating tumour vasculature and oxygenation $[51,52]$. Potential tumour physiological responses to exercise are shown in Fig. 1. However, mechanistic insight regarding the benefits of exercise on cancer biology is currently limited. Acute exercise bouts as well as exercise training are characterised by distinct physiological responses and result in specific immediate and chronic adaptations. It is well established that exercise leads to vascular adaptations in humans [53]. For example, exercise training improves oxygen delivery to peripheral tissues through 
vascular remodelling, namely angiogenesis and arteriogenesis $[54,55]$. The same structural remodelling of the vasculature in response to exercise may also take place in tumours, thus potentially improving oxygenation in the same way it does in working muscles during and after exercise. Moreover, under acute exercise conditions, mechanical forces exerted on vessel walls increase with increasing exercise intensity and are thought to promote the development of functional mature vasculature [56, 57]. Translated to dysfunctional tumour vasculature, exercise may result in the development of more mature and less permeable blood vessels that function more normally. Furthermore, increased heart rate and stroke volume during exercise result in augmented cardiac output and increase systemic blood flow, potentially also enhancing perfusion of tumour tissue. Together with increased tumour blood flow as a result of vasodilation mediated by mild hyperthermia that may occur during exercise and increases in mean arterial pressure that could result in an increased oxygen diffusion distance within the tumour, there are several potential exercise-related mechanisms that may alleviate tumour hypoxia and therefore enhance treatment response to radiotherapy (Fig. 2).

\section{Exercise modulation of tumour perfusion and hypoxia}

As detailed above, physical activity has been associated with a reduced risk of $\mathrm{PCa}$ progression and mortality [40, 41]. However, little is known about the underlying biological mechanisms that delay progression and improve survival in PCa patients. Potential mechanisms have been summarised by Galvão et al. [58] and include, among others, changes in tumour vascularisation that may permit better drug delivery and oxygenation of the tumour through improved perfusion [58]. Indeed, tumour vessel morphology has been associated with survival outcomes in men diagnosed with PCa. Mucci et al. [59] evaluated whether tumour microvessel morphology would predict $\mathrm{PCa}$ mortality among 572 men in the prostatectomy cohort of the Health Professionals Follow-up Study. While greater microvessel density (MVD) was associated with extraprostatic extension it was not a predictor of mortality in this analysis. However, the authors found that men with tumours containing the smallest (area and diameter) and most irregularly shaped vessels had a $\sim 6$ times and $\sim 17$ times higher likelihood of developing lethal PCa, respectively.

In a further investigation, also based on data from the Health Professionals Follow-up Study, Van Blarigan et al. [60] examined whether physical activity was associated with tumour vessel morphology in men with PCa. They found that men who reported a brisk walking pace 
of $\geq 4.8 \mathrm{~km} / \mathrm{h}$ had larger, more regularly shaped blood vessels compared to men who reported walking at $<4.8 \mathrm{~km} / \mathrm{h}$. However, there was no association between brisk walking and MVD, and the duration of physical activity (vigorous and non-vigorous) was not associated with tumour blood vessel size, shape or number [60].

In the following sections, we discuss the clinical and preclinical work that has been done to further shed light on potential mechanisms and how they might affect treatment outcomes. These sections are not intended to be an exhaustive review of the relevant literature that examine exercise as an adjunct therapy in cancer or a means to improve treatment outcomes. Rather, they are intended to provide an extensive overview of the efficacy of exercise to modulate tumour vasculature, perfusion and hypoxia as well as how these adaptations might translate to improved treatment outcomes. A literature search was conducted in Embase, MEDLINE and PubMed using the search terms exercise AND (cancer OR tumour) AND (perfusion OR blood flow OR hypoxia OR oxygen OR vascularity OR vascular OR vessels) as well as exercise AND prostate cancer AND (radiotherapy OR radiation), and last updated in February 2020.

\section{Preclinical studies}

Aerobic exercise has been linked to a reduction in tumour incidence, growth and metastasis across a variety of preclinical tumour models. However, meaningful comparisons and conclusions from current evidence is limited due to considerable heterogeneity in the methods used across different studies [61]. It has been proposed that exercise can directly impact tumour physiology and improve anti-cancer treatment efficacy, thus highlighting the potential of exercise to control disease progression [62]. One important mechanism by which exercise may govern these processes is modulation of tumour vascularisation and perfusion [63].

\section{Acute exercise responses}

Compared with short- as well as long-term exercise training interventions, few studies have investigated how an acute bout of exercise may affect tumour physiology (Table 1). Using a preclinical PCa model, McCullough et al. [64] published the first study to investigate tumour blood flow in vivo during exercise. The authors demonstrated that compared to resting conditions, exercise comprised of forced treadmill running significantly increased tumour blood flow by $\sim 200 \%$ as well as the number of perfused blood vessels, with hypoxia decreased by $\sim 50 \%$. These results were later replicated by Garcia et al. [65], who used a similar exercise protocol in a PCa model and demonstrated that exercise increased tumour blood flow by $\sim 180 \%$ compared to resting conditions. Interestingly, this response was only observed in orthotopic tumours mimicking the physiological conditions of primary cancers. Ectopic tumours, which could be regarded as metastatic lesions, showed a decrease in blood flow of $\sim 25 \%$ in response to exercise.

Miller et al. [66] and Schadler et al. [67] used radionuclide uptake and intratumoral doxorubicin levels as surrogate markers for tumour perfusion, respectively. Whereas Miller et al. [66] found an increased concentration of radiotracers with high-intensity exercise compared to a hypotensive low-perfusion state induced by anaesthesia, Schadler et al. [67] found no difference in doxorubicin levels after low-intensity exercise compared to mice not undergoing exercise. Despite exercising for more than twice the amount of time ( 45 vs. $20 \mathrm{~min})$, the mice in the experiment by Schadler et al. [67] only exercised at half the intensity (10 vs. $20 \mathrm{~m} / \mathrm{min}$ ), which likely resulted in overall lower mean arterial pressure compared to the experiment by Miller et al. [66] and therefore could have resulted in lower tumour perfusion pressure and hence lower tumour blood flow. Indeed, preliminary data from Lønbro et al. [68] suggest that it is only high-intensity exercise and not low- or moderate-intensity exercise that results in decreased intratumoral hypoxia.

The studies by McCullough et al. [64] and Garcia et al. [65] both also investigated in vitro vascular responsiveness to norepinephrine. In tumour arterioles, vasoconstriction was blunted by approximately $95 \%$ compared with control prostate vessels [64], and although there was no difference in vasoreactivity between orthotropic and ectopic tumours, vasoconstriction was severely reduced compared with host prostate tissue from healthy control animals [65]. This blunted vascular responsiveness is potentially what allows tumour perfusion to be either maintained or increased during exercise, when blood would otherwise normally be diverted away from internal organs to working muscles. Importantly, the resulting increase in tumour blood flow may play a critical role in improving oxygenation for radiotherapy as well as tumour-targeting drug delivery. Additionally, it has been proposed that acute systemic effects of exercise in combination with increased tumour perfusion may contribute to slower tumour progression [62]. However, it is currently unknown for how long after exercise these changes in tumour perfusion last and hence when the optimal time would be to exercise in relation to treatment, or at which point there is no added benefit of exercise. Furthermore, it is unclear what exercise prescription in terms of intensity and duration would provide the most potent stimulus that is both feasible and produces a clinically meaningful response. 


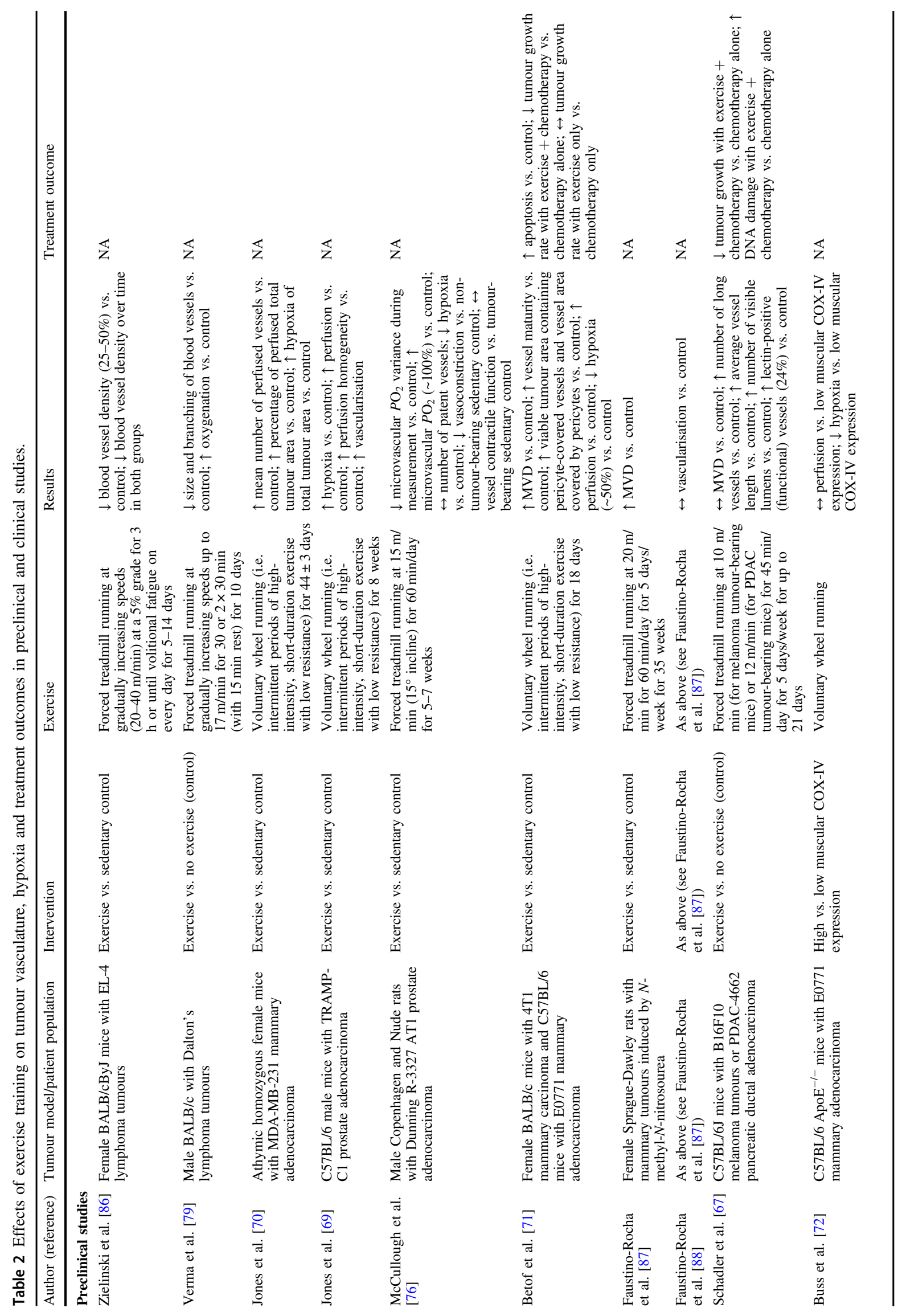




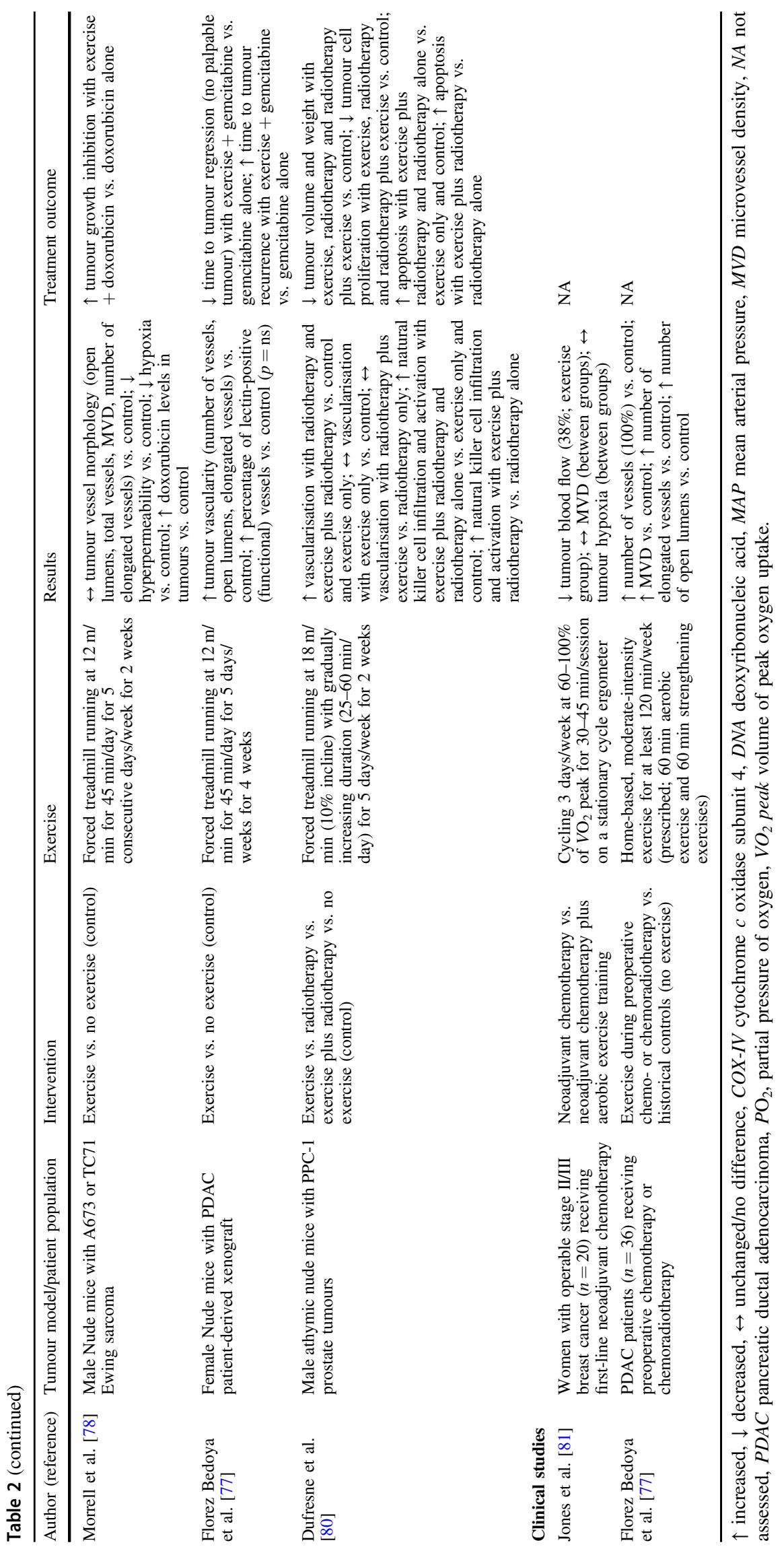




\section{Adaptations to exercise training}

Several animal model studies, predominantly in lymphoma, breast and $\mathrm{PCa}$, that investigated the effect of exercise training on tumour physiology, including vascular function and hypoxia, also assessed tumour growth. In studies by Jones et al. [69, 70], tumour growth rate was comparable between exercise groups who had voluntary access to a running wheel and non-exercising control groups. On the other hand, exercise significantly reduced tumour growth in a murine breast cancer model [71]. Buss and Dachs [72] also found that voluntary wheel running slowed tumour growth rate in hyperlipidemic mice. However, this was only the case for mice with high muscular cytochrome $c$ oxidase subunit IV (COX-IV) expression (a biomarker used for the level of exercise), suggesting that only mice with higher exercise volumes experienced a reduction in tumour growth rate [72]. Moreover, exercise appeared to suppress the formation and growth of metastases in exercising animals $[69,72]$. The anti-metastatic effect of exercise may be linked to its ability to alter vascular permeability through modulation of redox-sensitive small GTPase activity, thereby preventing cancer cells from easily crossing the vessel wall, making it more difficult to establish metastatic sites [73]. Indeed, studies by Wolff et al. showed that running distance was inversely correlated with activation of the redox-sensitive small GTPase Rho [74], and that exercise maintained blood-brain barrier integrity, thus preventing tumour cells from extravasating into the brain [75].

An overview of the studies investigating the impact of exercise training on tumour vasculature in an attempt to alleviate hypoxia and improve treatment response are presented in Table 2. Both short-term and long-term exercise training, in the form of voluntary wheel running or forced treadmill running, appear to improve intratumoral vasculature. However, while changes in vessel number and size as well as MVD are inconsistent, findings of increased vessel maturity and function that allow for tumours to be perfused more uniformly and thus increase microvascular $\mathrm{PO}_{2}$ are more conclusive. Schadler et al. [67] postulated that increased shear stress exerted on endothelial cells during aerobic exercise plays an important role in the remodelling of tumour blood vessels, allowing for greater blood flow to the tumour. In addition, McCullough et al. [76] found that temporal variations in microvascular $\mathrm{PO}_{2}$ were reduced in exercise-trained animals compared to sedentary control rats in an orthotopic PCa model. Studies have also shown that the number of patent and perfused vessels increased after exercise training, resulting in a larger percentage and less heterogeneously perfused tumour area [67, 69, 70, 77]. These changes in vascularisation and improvements in perfusion were accompanied by a reduction in tumour hypoxia [71, 72, 76, 78, 79], and mice with high COX-IV expression had significantly lower levels of hypoxia compared to mice with low levels of COX-IV expression [72].

\section{Effect of exercise on radiotherapy treatment response}

To date, only one study has investigated the effect of exercise in combination with radiotherapy on treatment outcomes [80]. Dufresne et al. injected human PCa PPC-1 cells subcutaneously into male athymic nude mice and subjected them to exercise 5 days/week for $25-60 \mathrm{~min} /$ day over 2 weeks in combination with a total of 20 Gray (Gy) fractionated into four doses of $5 \mathrm{~Gy}$ each. Mice were randomised to either no exercise (control), exercise only, radiotherapy only, or exercise plus radiotherapy. In contrast to previous studies, exercise alone did not increase tumour vascularisation; however, radiotherapy (with or without exercise) did. Radiotherapy further caused an increase in natural killer cell infiltration and activity that resulted in increased tumour cell apoptosis. Interestingly, this response was potentiated when exercise was combined with radiotherapy [80]. These results are exciting and provide preliminary preclinical evidence on the application of exercise as an adjunct therapy during radiotherapy in PCa.

\section{Clinical studies}

Despite the large number of clinical studies in the field of exercise oncology and although preclinical studies have produced promising results, very few clinical studies have actually investigated the effect of either an acute bout of exercise or prolonged exercise training on tumour vasculature, perfusion and hypoxia in humans. To our knowledge, only two studies have been conducted in a clinical population [77, 81] (Table 2). Jones et al. [81] performed a pilot study where they allocated 20 women with operable breast cancer receiving neoadjuvant chemotherapy to either chemotherapy alone or chemotherapy plus aerobic exercise training, consisting of thrice weekly cycling on a stationary ergometer at $60-100 \%$ of peak oxygen consumption for $30-45 \mathrm{~min} / \mathrm{session}$. At baseline and after 9 weeks of the intervention (i.e. after three cycles of chemotherapy), tumour blood flow was assessed using ${ }^{15} \mathrm{O}$-labelled water positron emission tomography (PET). In the exercise group, tumour blood flow decreased by an average of $38 \%$. However, the analysis was limited by the fact that pre/post PET scans were only available for five women in the exercise group (for two women in the control group for whom pre/post PET scans were available data were not reported). Further immunohistochemical analysis of tumour biopsies at the same time point (i.e. after 9 weeks of the intervention) revealed no difference between groups in relation to markers of hypoxia and MVD. However, similar to the PET assessment, tissue samples were only available 
for a small number of patients due to pathologic complete response rates to chemotherapy treatment.

In contrast, Florez Bedoya et al. [77] assessed tumour vessel morphology in tissue samples from 23 pancreatic ductal adenocarcinoma patients participating in an exercise intervention during preoperative chemo- or chemoradiation therapy and compared them to 13 historical controls (i.e. tumour samples from patients not undergoing exercise prior to surgery). The authors reported that $2 \mathrm{~h} /$ week of prescribed home-based exercise training resulted in remodelling of the tumour vasculature. Specifically, the total number of vessels, the number of elongated vessels, MVD as well as the number of open vessel lumens were all increased in samples from the exercise group compared with control samples. In theory, these vascular adaptations could result in improved chemotherapy and/or oxygen delivery to the tumour and hence improve clinical outcomes. However, further clinical studies are warranted to confirm these results and evaluate the effects of exerciseinduced vascular remodelling on treatment efficacy as well as long-term outcomes.

Neoadjuvant and pre-surgical settings provide an excellent opportunity to study some of these outcomes. Indeed, several clinical trials are currently ongoing or have recently been completed and are pending publication of results, which will add to this body of knowledge. The majority of these registered studies are being conducted in PCa patients, either investigating vascular adaptations in response to exercise training prior to prostatectomy and subsequently analysing resected tumour tissue samples for tumour vessel morphology and hypoxic tumour area as well as intratumoral natural killer cell infiltration (ClinicalTrials.gov Identifier: NCT02954783 and NCT03365076), or investigating changes in tumour perfusion and hypoxia in response to acute exercise exposure using either magnetic resonance imaging or by pathological analysis of tumour tissue samples excised during radical prostatectomy (ANZCTR Registration number: ACTRN12620000432910; ClinicalTrials.gov Identifier: NCT03675529). These studies will add further information regarding exercise as a potential modulator of the TME in clinical populations and provide insight into mechanistic pathways.

\section{Implications for future research}

Whereas initial clinical research shows mixed results, preclinical studies indicate that exercise may beneficially alter tumour physiology. In addition, one study in a PCa mouse model found that exercise increases the efficiency of radiotherapy, resulting in decreased tumour growth. These findings are promising and should encourage further investigation into this novel area of exercise and $\mathrm{PCa}$ research. However, given the paucity of research to date it is unclear what exercise prescription would deliver the desired outcome. Furthermore, specific tumour characteristics such as the initial degree of vascularity and metabolism will likely also influence outcome measures such as hypoxia and should be taken into consideration when designing future studies.

From a physiological perspective, the FITT principles of training, i.e. frequency, intensity, time (duration) and type of exercise should be considered as a starting point to formalise structured research questions. Different exercise intensities and types (e.g. aerobic vs. resistance training) are associated with distinct biochemical as well as physiological adaptations that may differentially affect tumour physiology. Furthermore, not all frequency schedules will likely be feasible for a specific exercise intensity (e.g. daily exercise at high intensity) and carefully considered periodisation schedules will need to be employed to maintain exercise tolerability during treatment [44, 82].

A concept that could be particularly interesting in this setting and patient population is the 'minimal dose approach' to exercise [83]. Considering the busy appointment schedule of patients, the minimal effective 'dose' of exercise (in terms of frequency, intensity and duration/time, or volume) would be extremely valuable to minimise patient burden. Hence, establishing or disproving dose-response relationships as well as threshold or ceiling effects will be essential to facilitate research translation into clinical practice. Similar to pharmaceutical drugs, exercise will likely also have a therapeutic window, where a certain minimal 'dose' is required to produce a desired effect and an upper tolerable 'dose' that will determine what is actually feasible for patients to safely accomplish.

In addition to the FITT principles of training, the timing of exercise in relation to treatment is likely to also be of importance. To achieve an oxygen enhancement effect for radiotherapy, the timing of exercise (i.e. pre-radiation) seems crucial, given that cellular oxygen is required at the time of radiation. Also, a distinction should be made between acute physiological changes and structural vascular adaptations that may aid treatment delivery and improve efficacy, including the time course in which these will likely occur.

From a clinical point of view, safety and efficacy trials in human populations are warranted. Although exercise is not a drug, it does drive a myriad of endogenous molecules to be produced that have influence locally and systemically as well as the effects of physical loading on vessels and other structures causing acute and chronic effects and adaptations. Therefore, it has been proposed that combining exercise with conventional cancer treatments should be held to the same standards as other combination treatments when evaluating treatment outcomes [84, 85]. Furthermore, 
logistical aspects that may affect the biological response also need to be taken into account. For example, studies in $\mathrm{PCa}$ patients investigating exercise in combination with radiotherapy may consider the timing and quantity of fluid intake before radiotherapy sessions, as gastrointestinal passage time and thus urine production may be affected by certain exercise durations and intensities. This may affect the positioning of the tumour mass due to fluctuating bladder volumes and ultimately lead to less radiation dose being delivered to the tumour and cause more toxicity to surrounding tissues.

Despite these potential challenges of implementing such trials, they will be a valuable addition to our understanding of the interaction between exercise and the TME and, if shown to be effective, will have clinical implications beyond the PCa population. However, men with PCa may particularly benefit from these developments, as initial observations appear to indicate that exercise of at least moderate intensity is required to elicit beneficial effects (i.e. increased tumour perfusion and subsequently reduced hypoxia). Men with $\mathrm{PCa}$ are usually relatively asymptomatic with respect to their disease and probably have a higher exercise capacity than many other cancer groups, such as pancreatic cancer patients, thus allowing them to exercise at an intensity to gain a benefit. Furthermore, men awaiting radical prostatectomy represent an ideal cohort to study these interactions as it allows researchers to directly study the impact of exercise on the tumour without the interference of neoadjuvant treatments and without having patients undergo additional invasive procedures to acquire tissue samples required to investigate potential mechanisms.

Collectively, further research in this area will add to the understanding of the underlying biological mechanisms of combination treatments involving exercise and address safety as well as dosing considerations important to both patients and clinicians. As a first important step, however, studies should address proof-of-concept outcomes such as short- and/or long-term reductions in tumour hypoxia as well as acute and chronic tumour perfusion changes as a result of exercise. We suggest these studies should incorporate non-invasive functional imaging modalities and immunohistochemical techniques to monitor tumour responses and pinpoint molecular mechanisms, with closely defined and reported patient populations and tumour characteristics as well as exercise prescription parameters to then develop more targeted interventions. Potentially, the findings of these studies will change best practice in radiation oncology with patients completing brief exercise bouts immediately (or shortly) before radiation doses, which may result in increased therapy success and a greater rate of cancer cure or delayed disease progression.
Acknowledgements OS is supported by a Ph.D. scholarship from the National Health and Medical Research Council Centre of Research Excellence in Prostate Cancer Survivorship.

\section{Compliance with ethical standards}

Conflict of interest The authors declare that they have no conflict of interest.

Publisher's note Springer Nature remains neutral with regard to jurisdictional claims in published maps and institutional affiliations.

Open Access This article is licensed under a Creative Commons Attribution 4.0 International License, which permits use, sharing, adaptation, distribution and reproduction in any medium or format, as long as you give appropriate credit to the original author(s) and the source, provide a link to the Creative Commons license, and indicate if changes were made. The images or other third party material in this article are included in the article's Creative Commons license, unless indicated otherwise in a credit line to the material. If material is not included in the article's Creative Commons license and your intended use is not permitted by statutory regulation or exceeds the permitted use, you will need to obtain permission directly from the copyright holder. To view a copy of this license, visit http://creativecommons. org/licenses/by/4.0/.

\section{References}

1. Delaney G, Jacob S, Featherstone C, Barton M. The role of radiotherapy in cancer treatment: estimating optimal utilization from a review of evidence-based clinical guidelines. Cancer. 2005;104:1129-37.

2. Skolarus TA, Wolf AM, Erb NL, Brooks DD, Rivers BM, Underwood W III, et al. American Cancer Society prostate cancer survivorship care guidelines. CA Cancer J Clin. 2014;64:225-49.

3. Barker HE, Paget JT, Khan AA, Harrington KJ. The tumour microenvironment after radiotherapy: mechanisms of resistance and recurrence. Nat Rev Cancer. 2015;15:409-25.

4. Kim BM, Hong Y, Lee S, Liu P, Lim JH, Lee YH, et al. Therapeutic implications for overcoming radiation resistance in cancer therapy. Int J Mol Sci. 2015;16:26880-913.

5. Vaupel P, Thews O, Höckel M. Treatment resistance of solid tumors: role of hypoxia and anemia. Med Oncol. 2001;18:243-59.

6. Cao L, Yang YJ, Li ZW, Wu HF, Yang ZC, Liu SX, et al. Moderate hypofractionated radiotherapy is more effective and safe for localized prostate cancer patients: a meta-analysis. Oncotarget. 2017;8:2647-58.

7. Coen JJ, Zietman AL, Thakral H, Shipley WU. Radical radiation for localized prostate cancer: local persistence of disease results in a late wave of metastases. J Clin Oncol. 2002;20:3199-205.

8. Zietman AL, DeSilvio ML, Slater JD, Rossi CJ Jr, Miller DW, Adams JA, et al. Comparison of conventional-dose vs high-dose conformal radiation therapy in clinically localized adenocarcinoma of the prostate: a randomized controlled trial. JAMA. 2005;294:1233-9.

9. Hughes VS, Wiggins JM, Siemann DW. Tumor oxygenation and cancer therapy-then and now. Br J Radiol. 2019;92:20170955.

10. Wilson WR, Hay MP. Targeting hypoxia in cancer therapy. Nat Rev Cancer. 2011;11:393-410.

11. Vaupel P, Kelleher DK. Blood flow and oxygenation status of prostate cancers. In: Advances in experimental medicine and 
biology, vol. 765. United States: Kluwer Academic/Plenum Publishers; 2013. p. 299-305.

12. Hayes BD, Brady L, Pollak M, Finn SP. Exercise and prostate cancer: evidence and proposed mechanisms for disease modification. Cancer Epidemiol Biomark Prev. 2016;25:1281-8.

13. Koelwyn GJ, Quail DF, Zhang X, White RM, Jones LW. Exercise-dependent regulation of the tumour microenvironment. Nat Rev Cancer. 2017;17:620-32.

14. Bloor CM. Angiogenesis during exercise and training. Angiogenesis. 2005;8:263-71.

15. Tonnesen MG, Feng X, Clark RA. Angiogenesis in wound healing. J Investig Dermatol Symp Proc. 2000;5:40-46.

16. Nagy JA, Chang SH, Dvorak AM, Dvorak HF. Why are tumour blood vessels abnormal and why is it important to know? $\mathrm{Br} \mathrm{J}$ Cancer. 2009;100:865-9.

17. Carmeliet P, Jain RK. Angiogenesis in cancer and other diseases. Nature. 2000;407:249-57.

18. Jain RK. Determinants of tumor blood flow: a review. Cancer Res. 1988;48:2641-58.

19. Chung AS, Lee J, Ferrara N. Targeting the tumour vasculature: insights from physiological angiogenesis. Nat Rev Cancer. 2010;10:505-14.

20. Sonveaux P. Provascular strategy: targeting functional adaptations of mature blood vessels in tumors to selectively influence the tumor vascular reactivity and improve cancer treatment. Radiother Oncol. 2008;86:300-13.

21. De Bock K, Mazzone M, Carmeliet P. Antiangiogenic therapy, hypoxia, and metastasis: risky liaisons, or not? Nat Rev Clin Oncol. 2011;8:393-404.

22. Horsman MR, Mortensen LS, Petersen JB, Busk M, Overgaard J. Imaging hypoxia to improve radiotherapy outcome. Nat Rev Clin Oncol. 2012;9:674-87.

23. Jordan BF, Sonveaux P. Targeting tumor perfusion and oxygenation to improve the outcome of anticancer therapy. Front Pharm. 2012;3:94.

24. Vaupel P, Harrison L. Tumor hypoxia: causative factors, compensatory mechanisms, and cellular response. Oncologist. 2004;9:4-9.

25. Vaupel P, Kelleher DK, Höckel M. Oxygenation status of malignant tumors: pathogenesis of hypoxia and significance for tumor therapy. Semin Oncol. 2001;28:29-35.

26. Gray LH, Conger AD, Ebert M, Hornsey S, Scott OC. The concentration of oxygen dissolved in tissues at the time of irradiation as a factor in radiotherapy. Br J Radiol. 1953;26:638-48.

27. Stewart GD, Ross JA, McLaren DB, Parker CC, Habib FK, Riddick AC. The relevance of a hypoxic tumour microenvironment in prostate cancer. BJU Int. 2010;105:8-13.

28. Milosevic M, Warde P, Menard C, Chung P, Toi A, Ishkanian A, et al. Tumor hypoxia predicts biochemical failure following radiotherapy for clinically localized prostate cancer. Clin Cancer Res. 2012;18:2108-14.

29. Vergis R, Corbishley CM, Norman AR, Bartlett J, Jhavar S, Borre $\mathrm{M}$, et al. Intrinsic markers of tumour hypoxia and angiogenesis in localised prostate cancer and outcome of radical treatment: a retrospective analysis of two randomised radiotherapy trials and one surgical cohort study. Lancet Oncol. 2008;9:342-51.

30. Jain RK. Antiangiogenesis strategies revisited: from starving tumors to alleviating hypoxia. Cancer Cell. 2014;26:605-22.

31. Martin JD, Seano G, Jain RK. Normalizing function of tumor vessels: progress, opportunities, and challenges. Annu Rev Physiol. 2019;81:505-34.

32. Elice F, Rodeghiero F. Side effects of anti-angiogenic drugs. Thromb Res. 2012;129:S50-53.

33. Elice F, Rodeghiero F, Falanga A, Rickles FR. Thrombosis associated with angiogenesis inhibitors. Best Pract Res Clin Haematol. 2009;22:115-28.
34. Jain RK. Normalization of tumor vasculature: an emerging concept in antiangiogenic therapy. Science. 2005;307:58-62.

35. Paez-Ribes M, Allen E, Hudock J, Takeda T, Okuyama H, Vinals $\mathrm{F}$, et al. Antiangiogenic therapy elicits malignant progression of tumors to increased local invasion and distant metastasis. Cancer Cell. 2009;15:220-31.

36. Oei AL, Kok HP, Oei SB, Horsman MR, Stalpers LJA, Franken NAP, et al. Molecular and biological rationale of hyperthermia as radio- and chemosensitizer. Adv Drug Deliv Rev. 2020. https:// doi.org/10.1016/j.addr.2020.01.003. [Epub ahead of print].

37. Cohen J, Anvari A, Samanta S, Poirier Y, Soman S, Alexander A, et al. Mild hyperthermia as a localized radiosensitizer for deepseated tumors: investigation in an orthotopic prostate cancer model in mice. Br J Radiol. 2019;92:20180759.

38. Caspersen CJ, Powell KE, Christenson GM. Physical activity, exercise, and physical fitness: definitions and distinctions for health-related research. Public Health Rep. 1985;100:126-31.

39. McTiernan A, Friedenreich CM, Katzmarzyk PT, Powell KE, Macko R, Buchner D, et al. Physical activity in cancer prevention and survival: a systematic review. Med Sci Sports Exerc. 2019;51:1252-61.

40. Kenfield SA, Stampfer MJ, Giovannucci E, Chan JM. Physical activity and survival after prostate cancer diagnosis in the Health Professionals Follow-up Study. J Clin Oncol. 2011;29:726-32.

41. Richman EL, Kenfield SA, Stampfer MJ, Paciorek A, Carroll PR, Chan JM. Physical activity after diagnosis and risk of prostate cancer progression: data from the Cancer of the Prostate Strategic Urologic Research Endeavor. Cancer Res. 2011;71:3889-95.

42. Courneya KS, Booth CM, Gill S, O'Brien P, Vardy J, Friedenreich CM, et al. The Colon Health and Life-Long Exercise Change trial: a randomized trial of the National Cancer Institute of Canada Clinical Trials Group. Curr Oncol. 2008;15:279-85.

43. Newton RU, Kenfield SA, Hart NH, Chan JM, Courneya KS, Catto $\mathrm{J}$, et al. Intense exercise for survival among men with metastatic castrate-resistant prostate cancer (INTERVAL-GAP4): a multicentre, randomised, controlled phase III study protocol. BMJ Open. 2018;8:e022899.

44. Hayes SC, Newton RU, Spence RR, Galvão DA. The Exercise and Sports Science Australia position statement: exercise medicine in cancer management. J Sci Med Sport. 2019;22:1175-99.

45. Buffart LM, Newton RU, Chinapaw MJ, Taaffe DR, Spry NA, Denham JW, et al. The effect, moderators, and mediators of resistance and aerobic exercise on health-related quality of life in older long-term survivors of prostate cancer. Cancer. 2015;121:2821-30.

46. Galvão DA, Spry N, Denham J, Taaffe DR, Cormie P, Joseph D, et al. A multicentre year-long randomised controlled trial of exercise training targeting physical functioning in men with prostate cancer previously treated with androgen suppression and radiation from TROG 03.04 RADAR. Eur Urol. 2014;65:856-64.

47. Galvão DA, Taaffe DR, Spry N, Joseph D, Newton RU. Combined resistance and aerobic exercise program reverses muscle loss in men undergoing androgen suppression therapy for prostate cancer without bone metastases: a randomized controlled trial. J Clin Oncol. 2010;28:340-7.

48. Segal RJ, Reid RD, Courneya KS, Sigal RJ, Kenny GP, Prud'Homme DG, et al. Randomized controlled trial of resistance or aerobic exercise in men receiving radiation therapy for prostate cancer. J Clin Oncol. 2009;27:344-51.

49. Cormie P, Galvão DA, Spry N, Joseph D, Chee R, Taaffe DR, et al. Can supervised exercise prevent treatment toxicity in patients with prostate cancer initiating androgendeprivation therapy: a randomised controlled trial. BJU Int. 2015;115:256-66.

50. Kapur G, Windsor PM, McCowan C. The effect of aerobic exercise on treatment-related acute toxicity in men receiving 
radical external beam radiotherapy for localised prostate cancer. Eur J Cancer Care. 2010;19:643-7.

51. Siemann DW, Horsman MR. Modulation of the tumor vasculature and oxygenation to improve therapy. Pharm Ther. 2015;153:107-24.

52. Wiggins JM, Opoku-Acheampong AB, Baumfalk DR, Siemann DW, Behnke BJ. Exercise and the tumor microenvironment: potential therapeutic implications. Exerc Sport Sci Rev. 2018;46:56-64.

53. Green DJ, Hopman MT, Padilla J, Laughlin MH, Thijssen DH. Vascular adaptation to exercise in humans: role of hemodynamic stimuli. Physiol Rev. 2017;97:495-528.

54. Prior BM, Lloyd PG, Yang HT, Terjung RL. Exercise-induced vascular remodeling. Exerc Sport Sci Rev. 2003;31:26-33.

55. Prior BM, Yang HT, Terjung RL. What makes vessels grow with exercise training? J Appl Physiol. 2004;97:1119-28.

56. Suh GY, Les AS, Tenforde AS, Shadden SC, Spilker RL, Yeung $\mathrm{JJ}$, et al. Hemodynamic changes quantified in abdominal aortic aneurysms with increasing exercise intensity using MR exercise imaging and image-based computational fluid dynamics. Ann Biomed Eng. 2011;39:2186-202.

57. Hahn C, Schwartz MA. Mechanotransduction in vascular physiology and atherogenesis. Nat Rev Mol Cell Biol. 2009; 10:53-62.

58. Galvão DA, Taaffe DR, Spry N, Gardiner RA, Taylor R, Risbridger GP, et al. Enhancing active surveillance of prostate cancer: the potential of exercise medicine. Nat Rev Urol. 2016;13:258-65.

59. Mucci LA, Powolny A, Giovannucci E, Liao Z, Kenfield SA, Shen R, et al. Prospective study of prostate tumor angiogenesis and cancer-specific mortality in the Health Professionals Followup Study. J Clin Oncol. 2009;27:5627-33.

60. Van Blarigan EL, Gerstenberger JP, Kenfield SA, Giovannucci EL, Stampfer MJ, Jones LW, et al. Physical activity and prostate tumor vessel morphology: data from the Health Professionals Follow-up Study. Cancer Prev Res. 2015;8:962-7.

61. Ashcraft KA, Peace RM, Betof AS, Dewhirst MW, Jones LW. Efficacy and mechanisms of aerobic exercise on cancer initiation, progression, and metastasis: a critical systematic review of in vivo preclinical data. Cancer Res. 2016;76:4032-50.

62. Hojman P, Gehl J, Christensen JF, Pedersen BK. Molecular mechanisms linking exercise to cancer prevention and treatment. Cell Metab. 2018;27:10-21.

63. Pedersen L, Christensen JF, Hojman P. Effects of exercise on tumor physiology and metabolism. Cancer J. 2015;21:111-6.

64. McCullough DJ, Stabley JN, Siemann DW, Behnke BJ. Modulation of blood flow, hypoxia, and vascular function in orthotopic prostate tumors during exercise. J Natl Cancer Inst. 2014;106: dju036.

65. Garcia E, Becker VG, McCullough DJ, Stabley JN, Gittemeier $\mathrm{EM}$, Opoku-Acheampong $\mathrm{AB}$, et al. Blood flow responses to mild-intensity exercise in ectopic vs. orthotopic prostate tumors; dependence upon host tissue hemodynamics and vascular reactivity. J Appl Physiol. 2016;121:15-24.

66. Miller A, Nace R, Ayala-Breton CC, Steele M, Bailey K, Peng $\mathrm{KW}$, et al. Perfusion pressure is a critical determinant of the intratumoral extravasation of oncolytic viruses. Mol Ther. 2016;24:306-17.

67. Schadler KL, Thomas NJ, Galie PA, Bhang DH, Roby KC, Addai $\mathrm{P}$, et al. Tumor vessel normalization after aerobic exercise enhances chemotherapeutic efficacy. Oncotarget. 2016;7:65429-40.

68. Lønbro S, Wittenborn T, Byrialsen Elming P, Horsman MR. High intensity treadmill running reduces tumour hypoxia in mice. Radiother Oncol. 2018;127:S592 (abstract PO-1055).

69. Jones LW, Antonelli J, Masko EM, Broadwater G, Lascola CD, Fels D, et al. Exercise modulation of the host-tumor interaction in an orthotopic model of murine prostate cancer. J Appl Physiol. 2012;113:263-72.

70. Jones LW, Viglianti BL, Tashjian JA, Kothadia SM, Keir ST, Freedland SJ, et al. Effect of aerobic exercise on tumor physiology in an animal model of human breast cancer. J Appl Physiol. 2010;108:343-8.

71. Betof AS, Lascola CD, Weitzel D, Landon C, Scarbrough PM, Devi GR, et al. Modulation of murine breast tumor vascularity, hypoxia and chemotherapeutic response by exercise. J Natl Cancer Inst. 2015;107:djv040.

72. Buss LA, Dachs GU. Voluntary exercise slows breast tumor establishment and reduces tumor hypoxia in $\mathrm{ApoE}(-/-)$ mice. $\mathrm{J}$ Appl Physiol. 2018;124:938-49.

73. Wolff G, Toborek M. Targeting the therapeutic effects of exercise on redox-sensitive mechanisms in the vascular endothelium during tumor progression. IUBMB Life. 2013;65:565-71.

74. Wolff G, Balke JE, Andras IE, Park M, Toborek M. Exercise modulates redox-sensitive small GTPase activity in the brain microvasculature in a model of brain metastasis formation. PLoS ONE. 2014;9:e97033.

75. Wolff G, Davidson SJ, Wrobel JK, Toborek M. Exercise maintains blood-brain barrier integrity during early stages of brain metastasis formation. Biochem Biophys Res Commun. 2015;463:811-7.

76. McCullough DJ, Nguyen LM, Siemann DW, Behnke BJ. Effects of exercise training on tumor hypoxia and vascular function in the rodent preclinical orthotopic prostate cancer model. J Appl Physiol. 2013;115:1846-54.

77. Florez Bedoya CA, Cardoso ACF, Parker N, Ngo-Huang A, Petzel MQ, Kim MP, et al. Exercise during preoperative therapy increases tumor vascularity in pancreatic tumor patients. Sci Rep. 2019;9:13966.

78. Morrell MBG, Alvarez-Florez C, Zhang A, Kleinerman ES, Savage H, Marmonti E, et al. Vascular modulation through exercise improves chemotherapy efficacy in Ewing sarcoma. Pediatr Blood Cancer. 2019;66:e27835.

79. Verma VK, Singh V, Singh MP, Singh SM. Effect of physical exercise on tumor growth regulating factors of tumor microenvironment: implications in exercise-dependent tumor growth retardation. Immunopharmacol Immunotoxicol. 2009;31:274-82.

80. Dufresne S, Gueritat J, Chiavassa S, Noblet C, Assi M, RiouxLeclercq $\mathrm{N}$, et al. Exercise training improves radiotherapy efficiency in a murine model of prostate cancer. FASEB J. 2020;34:4984-96.

81. Jones LW, Fels DR, West M, Allen JD, Broadwater G, Barry WT, et al. Modulation of circulating angiogenic factors and tumor biology by aerobic training in breast cancer patients receiving neoadjuvant chemotherapy. Cancer Prev Res. 2013;6:925-37.

82. Kirkham AA, Bland KA, Zucker DS, Bovard J, Shenkier T, McKenzie DC, et al. "Chemotherapy-periodized" exercise to accommodate for cyclical variation in fatigue. Med Sci Sports Exerc. 2020;52:278-86.

83. Fisher JP, Steele J, Gentil P, Giessing J, Westcott WL. A minimal dose approach to resistance training for the older adult; the prophylactic for aging. Exp Gerontol. 2017;99:80-86.

84. Jones LW. Precision oncology framework for investigation of exercise as treatment for cancer. J Clin Oncol. 2015;33:4134-7.

85. Iyengar NM, Jones LW. Development of exercise as interception therapy for cancer: a review. JAMA Oncol. 2019;5:1620-7.

86. Zielinski MR, Muenchow M, Wallig MA, Horn PL, Woods JA. Exercise delays allogeneic tumor growth and reduces intratumoral inflammation and vascularization. J Appl Physiol. 2004;96:2249-56. 
87. Faustino-Rocha AI, Silva A, Gabriel J, Gil da Costa RM, Moutinho M, Oliveira PA, et al. Long-term exercise training as a modulator of mammary cancer vascularization. Biomed Pharmacother. 2016;81:273-80.
88. Faustino-Rocha AI, Gama A, Oliveira PA, Vanderperren K, Saunders JH, Pires MJ, et al. A contrast-enhanced ultrasonographic study about the impact of long-term exercise training on mammary tumor vascularization. J Ultrasound Med. 2017;36:2459-66. 